\title{
Research Article \\ Optimal Signal Reconstruction Using the Empirical Mode Decomposition
}

\author{
Binwei Weng ${ }^{1}$ and Kenneth E. Barner ${ }^{2}$ \\ ${ }^{1}$ Philips Medical Systems, MS 455, Andover, MA 01810, USA \\ ${ }^{2}$ Department of Electrical and Computer Engineering, University of Delaware, Newark, DE 19716, USA
}

Correspondence should be addressed to Kenneth E. Barner, barner@ece.udel.edu

Received 26 August 2007; Revised 12 February 2008; Accepted 20 July 2008

Recommended by Nii O. Attoh-Okine

\begin{abstract}
The empirical mode decomposition (EMD) was recently proposed as a new time-frequency analysis tool for nonstationary and nonlinear signals. Although the EMD is able to find the intrinsic modes of a signal and is completely self-adaptive, it does not have any implication on reconstruction optimality. In some situations, when a specified optimality is desired for signal reconstruction, a more flexible scheme is required. We propose a modified method for signal reconstruction based on the EMD that enhances the capability of the EMD to meet a specified optimality criterion. The proposed reconstruction algorithm gives the best estimate of a given signal in the minimum mean square error sense. Two different formulations are proposed. The first formulation utilizes a linear weighting for the intrinsic mode functions (IMF). The second algorithm adopts a bidirectional weighting, namely, it not only uses weighting for IMF modes, but also exploits the correlations between samples in a specific window and carries out filtering of these samples. These two new EMD reconstruction methods enhance the capability of the traditional EMD reconstruction and are well suited for optimal signal recovery. Examples are given to show the applications of the proposed optimal EMD algorithms to simulated and real signals.
\end{abstract}

Copyright (C) 2008 B. Weng and K. E. Barner. This is an open access article distributed under the Creative Commons Attribution License, which permits unrestricted use, distribution, and reproduction in any medium, provided the original work is properly cited.

\section{INTRODUCTION}

The empirical mode decomposition (EMD) is proposed by Huang et al. as a new signal decomposition method for nonlinear and nonstationary signals [1]. It provides an alternative to traditional time-frequency or time-scale analysis methods, such as the short-time Fourier transform and wavelet analysis. The EMD decomposes a signal into a collection of oscillatory modes, called intrinsic mode functions (IMF), which represent fast to slow oscillations in the signal. Each IMF can be viewed as a subband of a signal. Therefore, the EMD can be viewed as a subband signal decomposition. Traditional signal analysis tools, such as Fourier or wavelet-based methods, require some predefined basis functions to represent a signal. The EMD relies on a fully data-driven mechanism that does not require any $a$ priori known basis. It has also been shown that the EMD has some relationship with wavelets and filterbank. It is reported that the EMD behaves as a "wavelet-like" dyadic filter bank for fractional Gaussian noise $[2,3]$. Due to these special properties, the EMD has been used to address many science and engineering problems [4-13]. Although the EMD is computed iteratively and does not possess an analytical form, some interesting attempts have been made recently to address its analytical behavior [14].

The EMD depends only on the data itself and is completely unsupervised. In addition, it satisfies the perfect reconstruction (PR) property as the sum of all the IMFs yields the original signal. In some situations, however, not all the IMFs are needed to obtain certain desired properties. For instance, when the EMD is used for denoising a signal, partial reconstruction based on the IMF energy eliminates noise components [15]. Such partial reconstruction utilizes a binary IMF decision, that is, either discarding or keeping IMFs in the partial summation. Such partial reconstruction is not based on any optimality conditions. In this paper, we give an optimal signal reconstruction method that utilizes differently weighted IMFs and IMF samples. Stated more formally, the problem addressed here is the following: given a signal, how best to reconstruct the signal by the IMFs 
obtained from a signal that bears some relationship to the given signal. This can be regarded as a signal approximation or reconstruction problem and is similar to the filtering problem in which an estimated signal is obtained by filtering a given signal. The problem arises in many applications such as signal denoising and interference cancellation. The optimality criterion used here is the mean square error. Numerous methodologies can be employed to combine the IMFs to form an estimate. A direct approach is using linear weighting of IMFs. This leads to our first proposed optimal signal reconstruction algorithm based on EMD (OSR-EMD). For notational brevity, the suffix EMD is omitted and OSR, BOSR, and RBOSR are used instead of OSR-EMD, BOSREMD, RBOSR-EMD. A second approach is using weighting coefficients along both vertical IMF index direction and horizontal temporal index direction. Because of this, the second approach is named as the bidirectional optimal signal reconstruction algorithm (BOSR-EMD). As a supplement to the BOSR, a regularized version of BOSR (RBOSR-EMD) is also proposed to overcome the numerical instability of the BOSR. Simulation examples show that the proposed algorithms are well suited for signal reconstruction and significantly improve the partial reconstruction EMD.

The structure of the paper is as follows. In Section 2, we give a brief introduction to the EMD. Then the OSR is formulated in Section 3. The BOSR and RBOSR algorithms are proposed in Section 4. Simulation examples are given in Section 5 to demonstrate the efficacy of the algorithms. Finally, conclusions are made in Section 6.

\section{EMPIRICAL MODE DECOMPOSITION}

The aim of the EMD is to decompose a signal into a sum of intrinsic mode functions (IMF). An IMF is defined as a function with equal number of extrema and zero crossings (or at most differed by one) with its envelopes, as defined by all the local maxima and minima, being symmetric with respect to zero [1]. An IMF represents a simple oscillatory mode as a counterpart to the simple harmonic function used in Fourier analysis.

Given a signal $x(n)$, the starting point of the EMD is the identification of all the local maxima and minima. All the local maxima are then connected by a cubic spline curve as the upper envelop $e_{u}(n)$. Similarly, all the local minima are connected by a spline curve as the lower envelop $e_{l}(n)$. The mean of the two envelops is denoted as $m_{1}(n)=\left[e_{u}(n)+\right.$ $\left.e_{l}(n)\right] / 2$ and subtracted from the signal. Thus the first protoIMF $h_{1}(n)$ is obtained as

$$
h_{1}(n)=x(n)-m_{1}(n)
$$

The above procedure to extract the IMF is referred to as the sifting process. Since $h_{1}(n)$ still contains multiple extrema between zero crossings, the sifting process is performed again on $h_{1}(n)$. This process is applied repetitively to the protoIMF $h_{k}(n)$ until the first IMF $c_{1}(n)$, which satisfies the IMF condition, is obtained. Some stopping criteria are used to

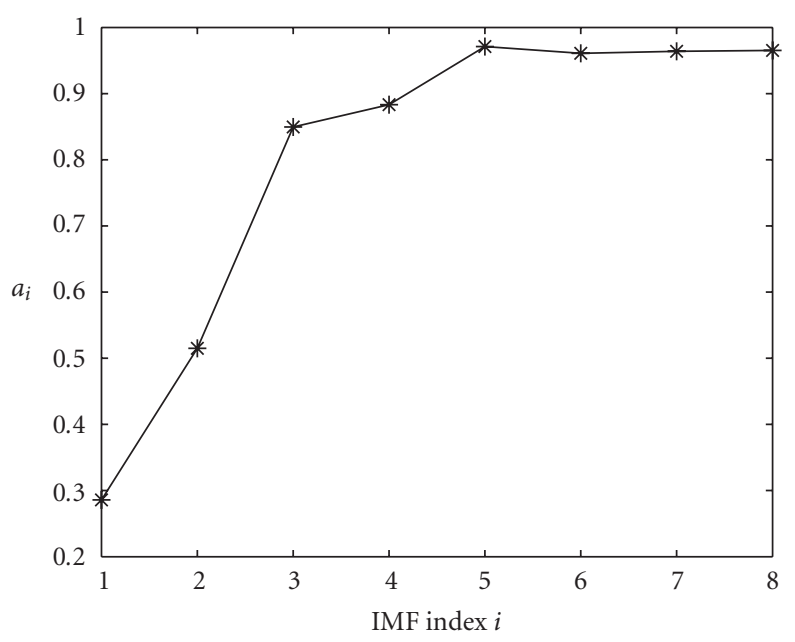

Figure 1: Optimal coefficients $a_{i}$ 's for the OSR.

terminate the sifting process. A commonly used criterion is the sum of difference (SD):

$$
\mathrm{SD}=\sum_{n=0}^{T} \frac{\left|h_{k-1}(n)-h_{k}(n)\right|^{2}}{h_{k-1}^{2}(n)}
$$

When the SD is smaller than a threshold, the first IMF $c_{1}(n)$ is obtained, which is written as

$$
r_{1}(n)=x(n)-c_{1}(n)
$$

Note that the residue $r_{1}(n)$ still contains some useful information. We can therefore treat the residue as a new signal and apply the above procedure to obtain

$$
\begin{gathered}
r_{1}(n)-c_{2}(n)=r_{2}(n) \\
\vdots \\
r_{N-1}(n)-c_{N}(n)=r_{N}(n) .
\end{gathered}
$$

The whole procedure terminates when the residue $r_{N}(n)$ is either a constant, a monotonic slope, or a function with only one extremum. Combining the equations in (3) and (4) yields the EMD of the original signal,

$$
x(n)=\sum_{i=1}^{N} c_{i}(n)+r_{N}(n) .
$$

The result of the EMD produces $N$ IMFs and a residue signal. For convenience, we refer to $c_{i}(n)$ as the $i$ th-order IMF. By this convention, lower order IMFs capture fast oscillation modes while higher order IMFs typically represent slow oscillation modes. If we interpret the EMD as a time-scale analysis method, lower-order IMFs and higher-order IMFs correspond to the fine and coarse scales, respectively. The residue itself can be regarded as the last IMF. 


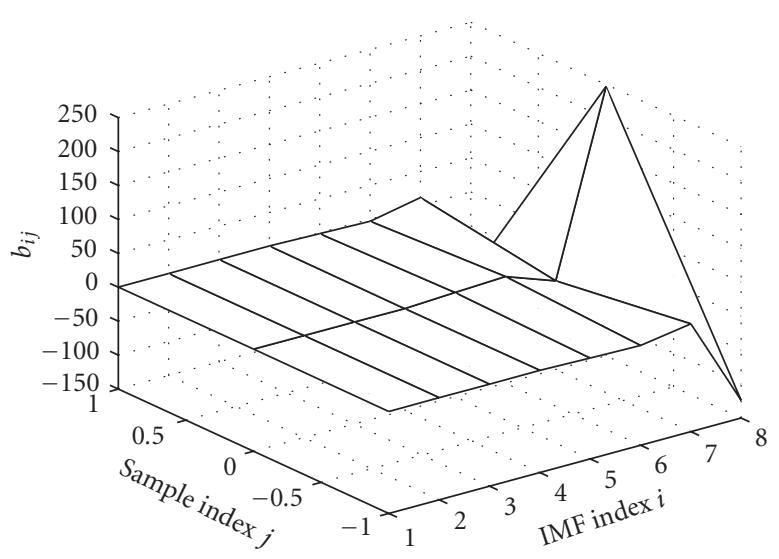

FIgURE 2: Optimal coefficients $b_{i j}$ 's for the BOSR.

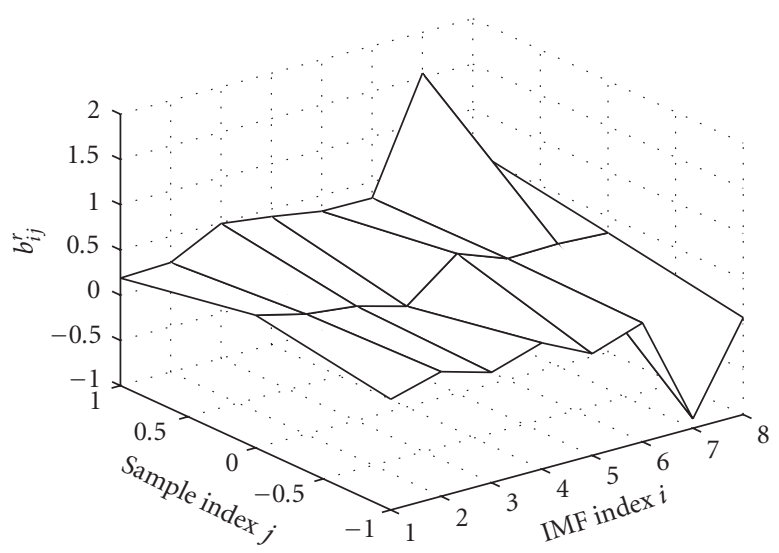

Figure 3: Optimal coefficients $b_{i j}$ 's for the RBOSR.

\section{OPTIMAL SIGNAL RECONSTRUCTION USING EMD}

The traditional empirical mode decomposition presented in the previous section is a perfect reconstruction (PR) decomposition as the sum of all IMFs yields the original signal. Consider the related problem in which the objective is to combine the IMFs in a fashion that approximates a signal $d(n)$ that is related to $x(n)$. This problem is exemplified by signal denoising application where $x(n)$ is a noise-corrupted version of $d(n)$ and the aim is to reconstruct $d(n)$ from $x(n)$. The IMFs can be combined utilizing various methodologies and under various objective functions designed to approximate $d(n)$. We consider several such methods beginning with a simple linear weighting,

$$
\widehat{d}(n)=\sum_{i=1}^{N} a_{i} c_{i}(n)
$$

where the coefficient $a_{i}$ is the weight assigned to the ith IMF. Note that, for convenience, the residue term is absorbed in the summation as the last term $c_{N}(n)$. Also, the IMFs are generated by decomposing $x(n)$, which has some relationship with the desired signal $d(n)$. To optimize the $a_{i}$ coefficients, we employ the mean square error (MSE),

$$
J_{1}=E\left\{[d(n)-\hat{d}(n)]^{2}\right\}=E\left\{\left[d(n)-\sum_{i=1}^{N} a_{i} c_{i}(n)\right]^{2}\right\} .
$$

The optimal coefficients can be determined by taking the derivative of (7) with respect to $a_{i}$ and setting it to zero. Therefore, we obtain

$$
\sum_{j=1}^{N} a_{j} E\left\{c_{i}(n) c_{j}(n)\right\}=E\left\{d(n) c_{i}(n)\right\},
$$

or equivalently,

$$
\sum_{i=1}^{N} R_{i j} a_{j}=p_{i}, \quad i=1, \ldots, N
$$

by defining

$$
p_{i}=E\left\{d(n) c_{i}(n)\right\}, \quad R_{i j}=E\left\{c_{i}(n) c_{j}(n)\right\} .
$$

The above $N$ equations can be written in a matrix form as follows:

$$
\left[\begin{array}{cccc}
R_{11} & R_{12} & \cdots & R_{1 N} \\
R_{21} & R_{22} & \cdots & R_{2 N} \\
\vdots & \vdots & \ddots & \vdots \\
R_{N 1} & R_{N 2} & \cdots & R_{N N}
\end{array}\right]\left[\begin{array}{c}
a_{1} \\
a_{2} \\
\vdots \\
a_{N}
\end{array}\right]=\left[\begin{array}{c}
p_{1} \\
p_{2} \\
\vdots \\
p_{N}
\end{array}\right]
$$

which can be compactly written as

$$
\mathbf{R}_{1} \mathbf{a}=\mathbf{p}
$$

The optimal coefficients are thus given by

$$
\mathbf{a}^{*}=\mathbf{R}_{1}^{-1} \mathbf{p}
$$

The dimension of the matrix $\mathbf{R}_{1}$ is $N \times N$. Since the number of IMFs $N$ is usually a small integer number, the matrix inversion does not incur any numerical difficulties. The minimum MSE can also be found by substituting (13) into (7), which yields

$$
J_{1, \min }=E\left\{\left[d(n)-\sum_{i=1}^{N} a_{i}^{*} c_{i}(n)\right]^{2}\right\}=\sigma_{d}^{2}-\mathbf{p}^{T} \mathbf{R}_{1}^{-1} \mathbf{p},
$$

where $\sigma_{d}^{2}=E\left\{d^{2}(n)\right\}$ is the variance of the desired signal. In practice, $p_{i}$ and $R_{i j}$ are estimated by sample average.

Many signals to which the EMD is applied are nonstationary. Also matrix inversion may be too costly in some situations. In such cases, an iterative gradient descent adaptive approach can be utilized:

$$
a_{i}(n+1)=a_{i}(n)-\left.\mu \frac{\partial J_{1}}{\partial a_{i}}\right|_{a_{i}=a_{i}(n)},
$$




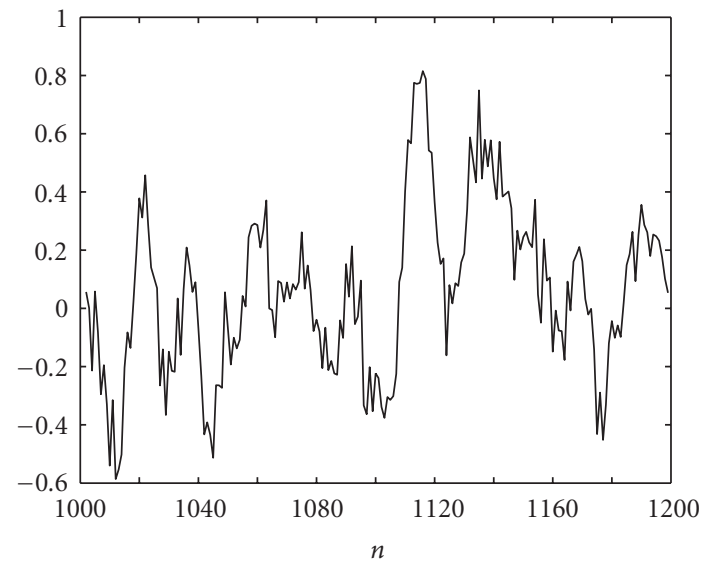

(a)

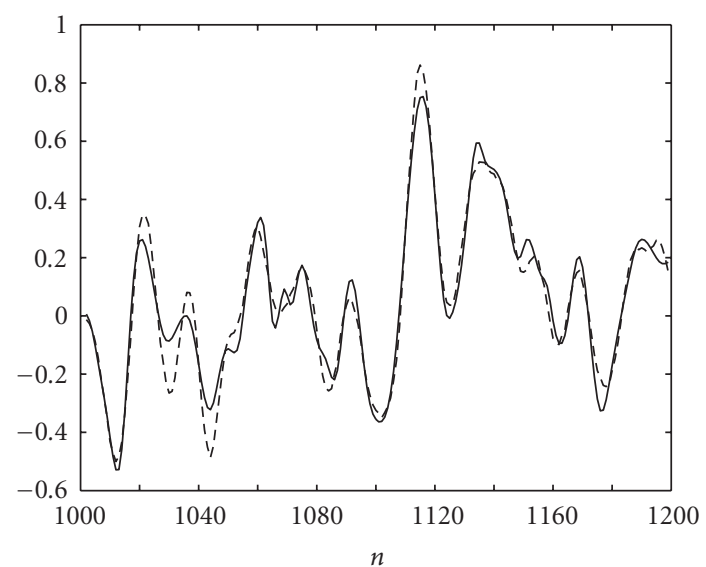

- - - Original

— PAR-EMD

(c)

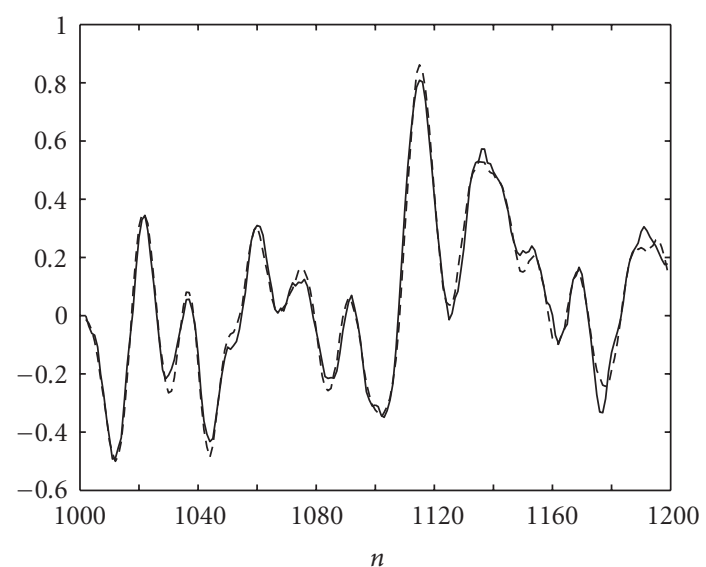

- - Original

— BOSR

(e)

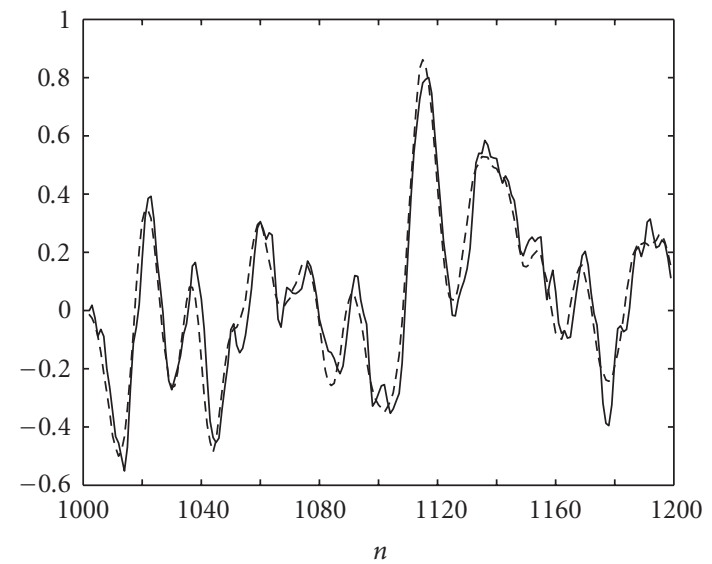

- - Original

— Linear filter

(b)

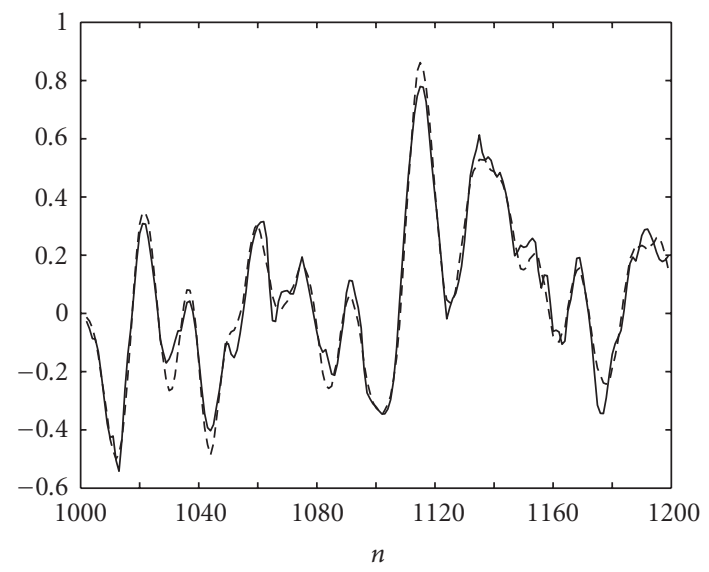

- - Original

_ OSR

(d)

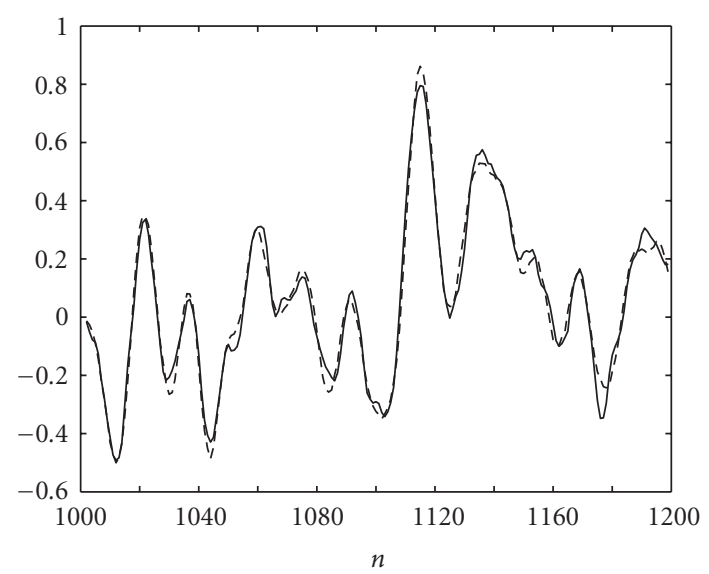

- - Original

- RBOSR

FIGURE 4: Denoising performance. Shown in dash lines are the original signal and the solid lines are denoised signals. (a) Noisy signal, (b) linear Butterworth filter, (c) PAR-EMD, (d) OSR, (e) BOSR, (f) RBOSR. 


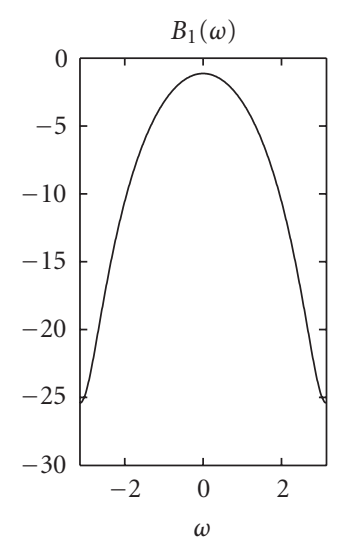

(a)

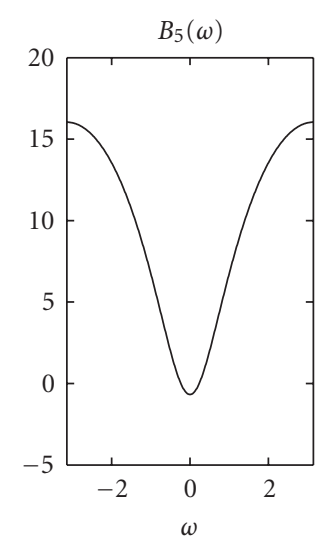

(e)

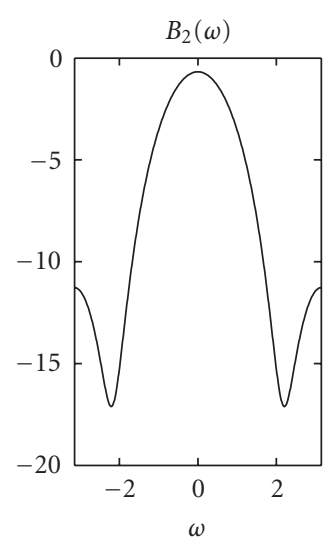

(b)

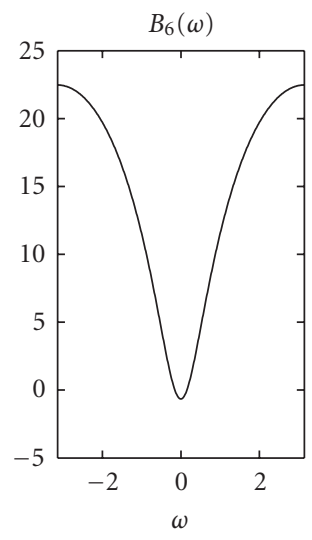

(f)

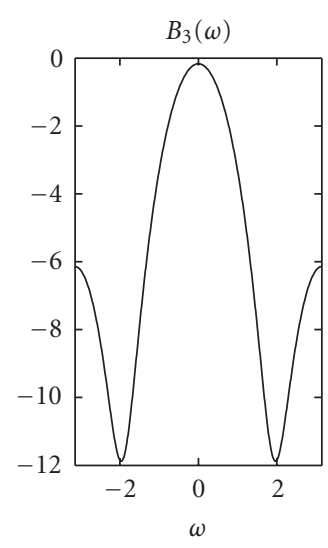

(c)

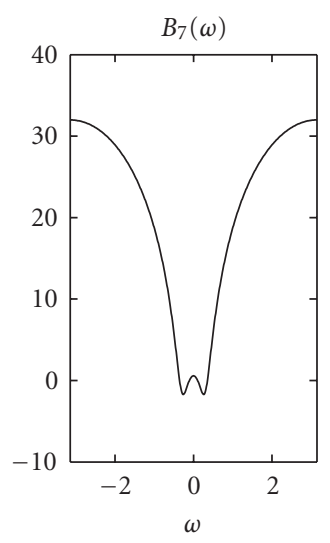

(g)

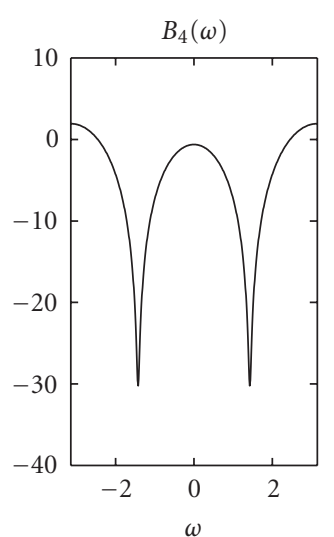

(d)

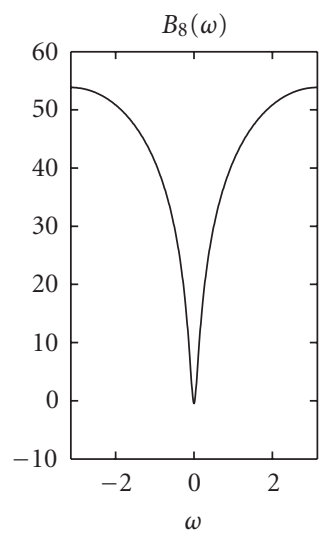

(h)

Figure 5: Equivalent filter frequency responses for BOSR algorithm coefficients. Frequency responses of $B_{1}-B_{8}$ are shown in dB values.

where $\mu$ is a positive number controlling the convergence speed. By taking the gradient and using instantaneous estimate for expectation, we obtain

$$
\begin{aligned}
\frac{\partial J_{1}}{\partial a_{i}} & =-2 E\left\{\left[d(n)-\sum_{i=1}^{N} a_{i}(n) c_{i}(n)\right] c_{i}(n)\right\} \\
& =-2 E\left\{e(n) c_{i}(n)\right\} \approx-2 e(n) c_{i}(n) .
\end{aligned}
$$

Therefore, the weight update equation (15) can be written as

$$
a_{i}(n+1)=a_{i}(n)+2 \mu e(n) c_{i}(n), \quad i=1, \ldots, N
$$

From the above formulation, it is clear that the OSR is very similar to the Wiener filtering, which aims to estimate a desired signal by passing a signal through a linear filter. The main difference is that the OSR operates samples in the EMD domain and weights samples according to the IMF order while the Wiener filter applies filtering to time domain signals directly and weights them temporally. Two special cases of the OSR are remarked as follows. If all the coefficients $a_{i}=1$, then it is equivalent to the original perfect reconstruction EMD (PR-EMD). If some coefficients are set to zero while others are set to one, it reduces to the partial reconstruction EMD (PAR-EMD) used in $[8,15]$. Therefore, the OSR extends the capability of the traditional EMD reconstruction and more importantly, yields the optimal estimate of a given signal in the mean square error sense.

\section{BIDIRECTIONAL OPTIMAL SIGNAL RECONSTRUCTION USING EMD}

In the EMD, there are two directions in the resulting IMFs. The first direction is the vertical direction denoted by the IMF order $i$ in (5). The vertical direction corresponds to different scales. The other direction is the horizontal direction represented by the time index $n$ in (5). This direction captures the time evolution of the signal. The OSR proposed in the last section only uses the weighting along the vertical direction. Therefore, it lacks degree of freedom in the horizontal, or temporal direction. In some circumstances, adjacent signal samples are correlated and this factor must be considered when performing reconstruction.

A more flexible EMD reconstruction algorithm that incorporates the signal correlation among samples in a 


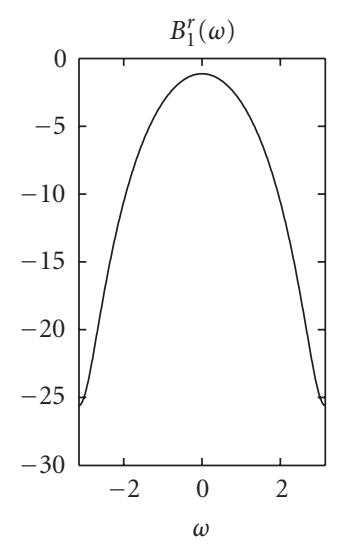

(a)

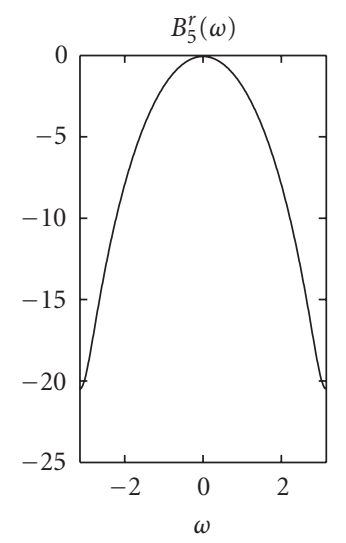

(e)

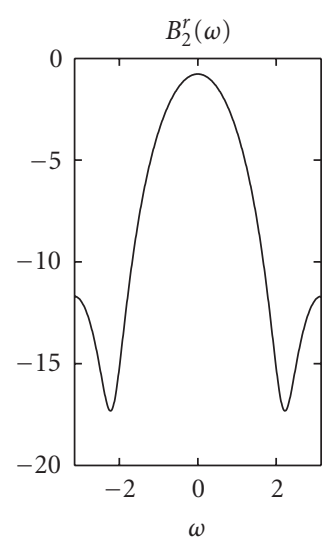

(b)

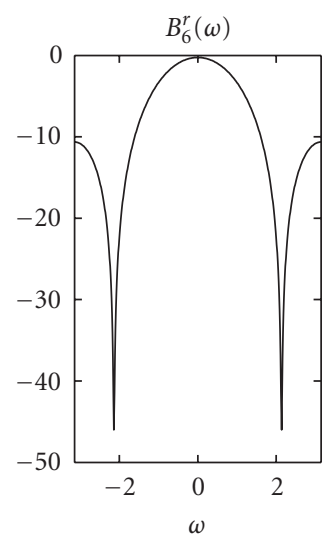

(f)

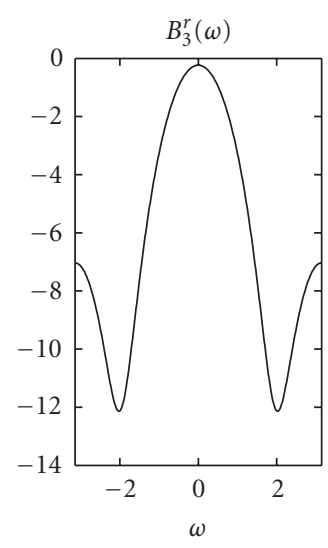

(c)

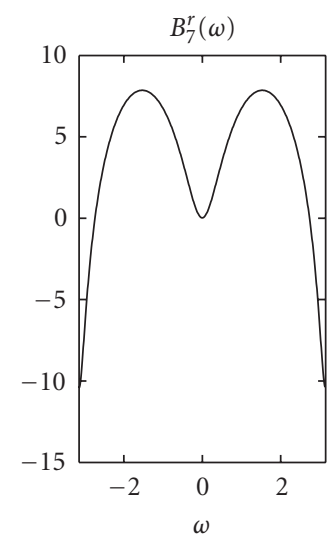

(g)

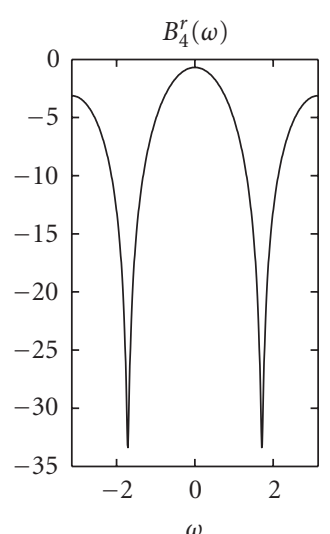

(d)

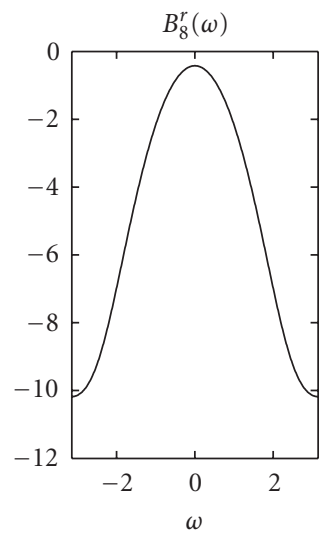

(h)

FIGURE 6: Equivalent filter frequency responses for RBOSR algorithm coefficients. Frequency responses of $B_{1}-B_{8}$ are shown in dB values.

temporal window is described as follows. For a specific time $n$, a temporal window of size $2 M+1$ is chosen with the current sample being the center of the window. Weighting is concurrently employed to account for the relations between IMFs. Consequently, 2D weighting coefficients $b_{i j}$ are utilized to yield the estimated signal

$$
\hat{d}(n)=\sum_{i=1}^{N} \sum_{j=-M}^{M} b_{i j} c_{i}(n-j)
$$

where $M$ is the half window length. This formulation takes both vertical and horizontal directions into consideration and is thus referred to as the bidirectional optimal signal reconstruction (BOSR). From (18), the bidirectional weighting can be interpreted as follows. The $i$ th IMF $c_{i}(n)$ is passed through a FIR filter $b_{i j}$ of length $2 M+1$. Thus we have a filter bank consisting of $N$ FIR filters, each of which is applied to an individual IMF. The final output is the summation of all filter outputs. Compared to the OSR, the BOSR makes use of the correlation between the samples. However, the cost paid for the gained degrees of freedom is increased computational complexity.
Similar to the OSR, the optimization criterion chosen here is the mean square error

$$
J_{2}=E\left\{\left[d(n)-\sum_{i=1}^{N} \sum_{j=-M}^{M} b_{i j} c_{i}(n-j)\right]^{2}\right\}
$$

Differentiating, with respect to the coefficient $b_{i j}$ and setting it to zero, yields

$$
\begin{gathered}
\sum_{k=1}^{N} \sum_{l=-M}^{M} b_{k l} R_{2}(k, i ; l, j)=p_{2}(i, j), \\
i=1, \ldots, N, \quad j=-M, \ldots, M,
\end{gathered}
$$

where we define

$$
\begin{gathered}
R_{2}(k, i ; l, j)=E\left\{c_{k}(n-l) c_{i}(n-j)\right\}, \\
p_{2}(i, j)=E\left\{d(n) c_{i}(n-j)\right\} .
\end{gathered}
$$

It can be seen that the correlation in (21) is bidirectional with a quadruple index representing both IMF order and 
temporal directions. There are altogether $(2 M+1) N$ equations in (20) and if we rearrange the $R_{2}(k, i ; l, j)$ and $p_{2}(i, j)$ according to the lexicographic order, (20) can be put into the following matrix equation:

$$
\left[\begin{array}{cccc}
R_{2}(1,1 ;-M,-M) & R_{2}(1,1 ;-M+1,-M) & \cdots & R_{2}(N, 1 ; M,-M) \\
R_{2}(1,1 ;-M,-M+1) & R_{2}(1,1 ;-M+1,-M+1) & \cdots & R_{2}(N, 1 ; M,-M+1) \\
\vdots & \vdots & \ddots & \vdots \\
R_{2}(1, N ;-M, M) & R_{2}(1, N ;-M+1, M) & \cdots & R_{2}(N, N ; M, M)
\end{array}\right]\left[\begin{array}{c}
b_{1,-M} \\
b_{1,-M+1} \\
\vdots \\
b_{N, M}
\end{array}\right]=\left[\begin{array}{c}
p_{2}(1,-M) \\
p_{2}(1,-M+1) \\
\vdots \\
p_{2}(N, M)
\end{array}\right]
$$

Equation (23) can be compactly written as

$$
\mathbf{R}_{2} \mathbf{b}=\mathbf{p}_{2},
$$

from which the optimal solution $\mathbf{b}^{*}$ is given by

$$
\mathbf{b}^{*}=\mathbf{R}_{2}^{-1} \mathbf{p}_{2} \text {. }
$$

The dimension of the matrix $\mathbf{R}_{2}$ is $(2 M+1) N \times(2 M+1) N$, so the computational complexity due to matrix inversion is increased from $\mathcal{O}\left(N^{3}\right)$ for the OSR algorithm to $\mathcal{O}((2 M+$ $\left.1)^{3} N^{3}\right)$. However, since the BOSR performs weighting in IMF order and temporal directions, it can better capture signal correlations. The elements of the matrix $\mathbf{R}_{2}$ and the vector $\mathbf{p}$ can be estimated by sample averages. As in the OSR case, an adaptive approach can be utilized. After some derivation, we obtain the weight update equation for BOSR:

$$
\begin{gathered}
b_{i j}(n+1)=b_{i j}(n)+2 \mu e(n) c_{i}(n-j), \\
i=1, \ldots, N, \quad j=-M \ldots, M .
\end{gathered}
$$

In the BOSR, the memory length $M$ needs to be chosen. More samples in the window will improve the performance as more signal memories are taken into consideration to account for the temporal correlation. However, the performance gain is no longer substantial when $M$ is increased to a certain number. As such, we can set up an objective function similar to Akaike information criterion (AIC) to determine the optimal memory length $M$ [16]. This process is analogous to choosing model order in the statistical modeling.

\subsection{Regularized bidirectional optimal signal reconstruction using EMD}

Although the BOSR considers the time domain correlations between samples, a problem arises in calculating the optimal coefficients $\mathbf{b}^{*}$ by (25), as the matrix $\mathbf{R}_{2}$ is sometimes ill conditioned.

To see why $\mathbf{R}_{2}$ is sometimes ill conditioned, let $\mathbf{R}_{2}=$ $E\left\{\mathbf{c}(n) \mathbf{c}^{T}(n)\right\}$ where

$$
\begin{aligned}
\mathbf{c}(n)=[ & c_{1}(n+M), \ldots, c_{1}(n-M), c_{2}(n+M), \ldots, \\
& \left.c_{2}(n-M), \ldots, c_{N}(n+M), \ldots, c_{N}(n-M)\right]^{T} .
\end{aligned}
$$

Also denote $\mathbf{R}_{2}(:, k)$ as the $k$ th column of the matrix $\mathbf{R}_{2}$. It can be shown that

$$
\mathbf{R}_{2}(:, k)=E\left\{\mathbf{c}(n) c_{i}(n-j)\right\}
$$

where $k=(i-1) \times(2 M+1)+j+M+1$ for $i=1, \ldots, N$, $j=-M, \ldots, M$. Note that when the IMF order $i$ is large, $c_{i}(n)$ tends to have fewer oscillations and thus fewer changes between consecutive samples. The extreme case is a nearly constant residue for the last $\operatorname{IMF} c_{N}(n)$. Thus, $c_{i}(n)$ becomes smoother when the order $i$ becomes large. Due to this fact, $c_{i}(n-j)$ and $c_{i}(n-j+1)$ are very similar for large $i$. Consequently, the two columns $\mathbf{R}_{2}(:, k)$ and $\mathbf{R}_{2}(:, k+1)$ are also very similar, which results in $\mathbf{R}_{2}$ being ill conditioned.

To alleviate the potential ill-condition problem of the BOSR, we propose a regularized version of the BOSR (RBOSR). The original objective function $J_{2}$ does not place any constraints on the $b_{i j}$ coefficients. We add some regularizing conditions on $b_{i j}$ by restricting their values to be in the range $-U \leq b_{i j} \leq U$. This condition implies that the magnitudes of the coefficients are bounded by a constant u.

The original problem is thus changed into the following constrained optimization problem:

$$
\begin{gathered}
\operatorname{minimize} \quad J_{2}=E\left\{\left[d(n)-\sum_{i=1}^{N} \sum_{j=-M}^{M} b_{i j} c_{i}(n-j)\right]^{2}\right\} \\
\text { subject to }-U \leq b_{i j} \leq \mathcal{U}, \quad \forall 1 \leq i \leq N, \quad-M \leq j \leq M .
\end{gathered}
$$

To solve the above constrained optimization problem, we can invoke the Kuhn-Tucker condition [17], which gives a necessary condition for the optimal solution. The Lagrangian of the minimization problem can be written as

$$
\begin{aligned}
& L\left(b_{i j}, \mu_{i j}, \lambda_{i j}\right) \\
& \quad=J_{2}\left(b_{i j}\right)+\sum_{i=1}^{N} \sum_{j=-M}^{M} \mu_{i j}\left(-b_{i j}-u\right)+\sum_{i=1}^{N} \sum_{j=-M}^{M} \lambda_{i j}\left(b_{i j}-u\right) .
\end{aligned}
$$

Applying the Kuhn-Tucker condition yields the following equations:

$$
\begin{gathered}
\nabla L\left(b_{i j}, \mu_{i j}, \lambda_{i j}\right)=\frac{\partial J_{2}}{\partial b_{i j}}-\mu_{i j}+\lambda_{i j}=0, \\
\mu_{i j}\left(-b_{i j}-\mathcal{u}\right)=0, \\
\lambda_{i j}\left(b_{i j}-u\right)=0 \\
\mu_{i j} \geq 0 \\
\lambda_{i j} \geq 0 .
\end{gathered}
$$




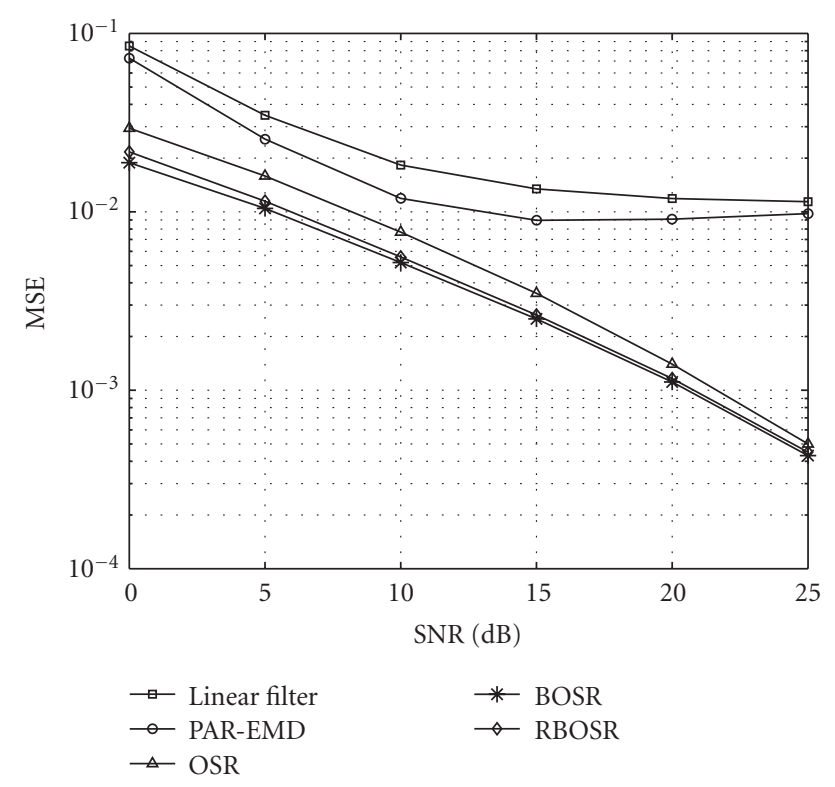

FIGURE 7: MSE versus SNR for three different denoising algorithms.

Iterative algorithms for general nonlinear optimization, such as the interior point method, can be utilized to find the optimal solution to the above problem [17]. A fundamental point of note is that the solution is guaranteed to be globally optimal since both the objective function and constraints are convex functions.

An alternative approach to solve the constrained minimization problem is to view it as a quadratic programming problem. The objective function can be rewritten as

$$
\begin{aligned}
J_{2} & =E\left\{\left[d(n)-\sum_{i=1}^{N} \sum_{j=-M}^{M} b_{i j} c_{i}(n-j)\right]^{2}\right\} \\
& =E\left\{d^{2}(n)\right\}-2 \mathbf{b}^{T} \mathbf{p}_{2}+\mathbf{b}^{T} \mathbf{R}_{2} \mathbf{b},
\end{aligned}
$$

where $\mathbf{b}, \mathbf{p}_{2}, \mathbf{R}_{2}$ are defined as in (24), and $\mathbf{c}(n)$ is the vector in (27). The optimization problem can thus be restated as a standard quadratic programming problem:

$$
\begin{gathered}
\operatorname{minimize} \quad J_{2}^{\prime}=\mathbf{b}^{T} \mathbf{R}_{2} \mathbf{b}-2 \mathbf{p}_{2}^{T} \mathbf{b} \\
\text { subject to } \quad-\boldsymbol{U} \preccurlyeq \mathbf{b} \preccurlyeq \boldsymbol{U},
\end{gathered}
$$

where the symbol $\preccurlyeq$ denotes component-wise less than or equal to for vectors. Since the objective function is convex and the inequality constraints are simple bounds, a faster conjugate gradient search for quadratic programming can be performed to find the optimal solution [17].

\section{APPLICATIONS}

Having established the OSR and BOSR algorithms, we apply them to various applications. Two examples are given. The first application considered is signal denoising, where simulated random signals are used. In the second example, the proposed algorithms are applied to real biomedical signals

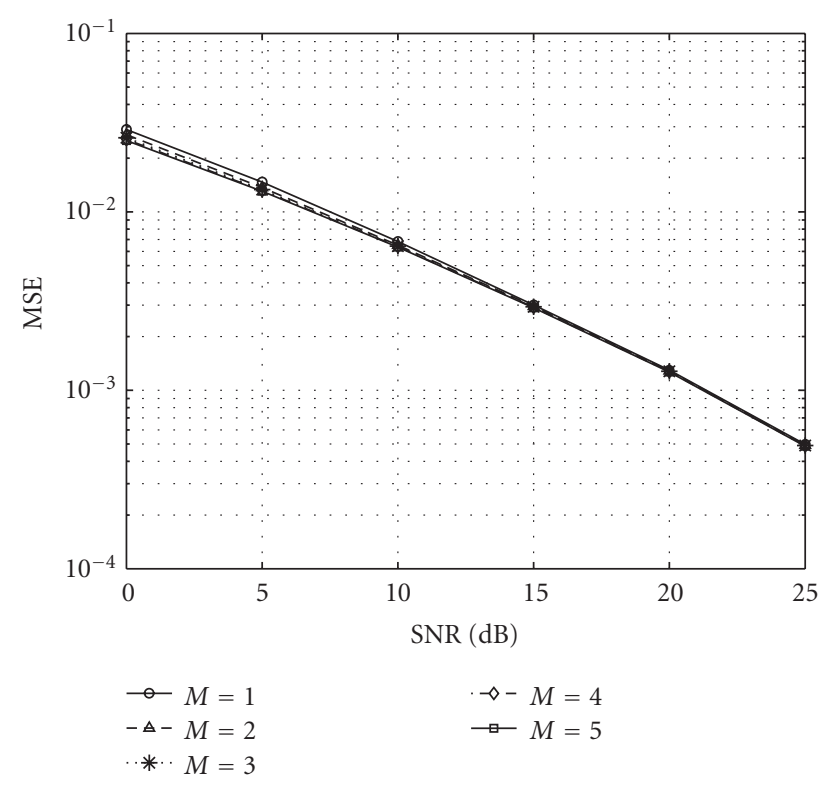

(a)

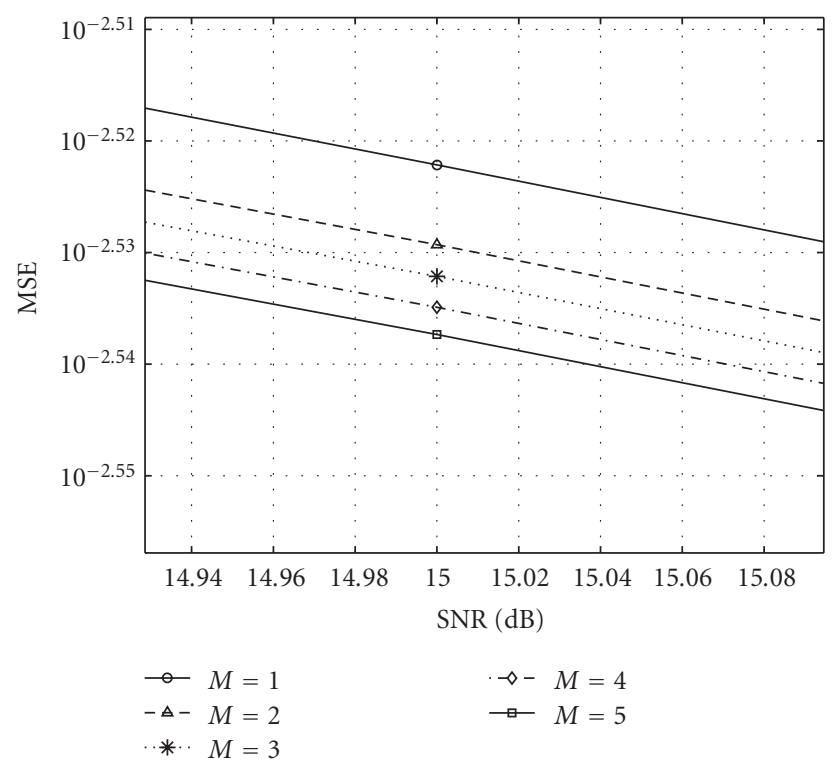

(b)

Figure 8: Performances for different memory length. (a) Largescale view, (b) zoomed-in view.

to remove ECG interferences from EEG recording. The following example illustrates the denoising using the OSR, BOSR, and RBOSR algorithms and compares them with the linear lowpass filtering and the partial reconstruction EMD (PAR-EMD) in [15]. The PAR-EMD method is based on the IMF signal energy and the reconstructed signal is given by the partial summation of those IMFs whose energy exceeds an established threshold.

Example 1. The original signal in this example is a bilinear signal model:

$$
x(n)=0.5 x(n-1)+0.6 x(n-1) v(n-1)+v(n),
$$


TABLE 1: Optimal coefficients of the OSR algorithm.

\begin{tabular}{lcccccccc}
\hline IMF order $i$ & 1 & 2 & 3 & 4 & 5 & 6 & 7 & 8 \\
\hline$a_{i}^{*}$ & 0.2859 & 0.5150 & 0.8496 & 0.8833 & 0.9710 & 0.9609 & 0.9639 & 0.9653 \\
\hline
\end{tabular}

TABLE 2: Optimal coefficients of the BOSR algorithm $(M=1)$.

\begin{tabular}{lcccccccc}
\hline$b_{i j}^{*}$ & & \multicolumn{7}{c}{ IMF order $i$} \\
& 1 & 2 & 3 & 4 & 5 & 6 & 7 \\
\hline-1 & 0.2219 & 0.3843 & 0.2322 & 0.5301 & -1.4488 & -3.0298 & 8.7950 & -123.9047 \\
0 & 0.4654 & 0.3261 & 0.2439 & -0.1592 & 3.6319 & 7.1100 & -19.3460 & 246.7700 \\
1 & 0.1899 & 0.2149 & 0.5048 & 0.5612 & -1.2579 & -3.1527 & 11.6191 & -121.9207 \\
\hline
\end{tabular}

where $v(n)$ is white noise with variance equal to 0.01 . Bilinear signal model is a type of nonlinear signal model. Additive Laplacian noise with variance 0.0092 is added to the signal to attain a SNR $=10 \mathrm{~dB}$, where SNR is defined as the ratio of signal power and noise variance. The total signal length is 2000 and the first 1000 samples are used as the training signal $d(n)$ to estimate the optimal OSR, BOSR, and RBOSR coefficients. Once these coefficients are determined, the remaining samples are tested for denosing. The denoised signal is obtained by substituting the optimal coefficients into the reconstruction formulae (6) and (18). In the following, the denoising performance is evaluated by the mean square error calculated as

$$
\mathrm{MSE}=\frac{1}{L_{2}-L_{1}+1} \sum_{n=L_{1}}^{L_{2}}\left[x_{o}(n)-\hat{x}(n)\right]^{2}
$$

where $L_{1}$ and $L_{2}$ are starting and ending indices of testing samples, and $x_{o}(n)$ and $\hat{x}(n)$ are original noise-free and denoised signals, respectively.

In the following, the signal memory $M$ in the BOSR is chosen to be 1. Eight IMFs are obtained after the EMD decomposition. Hence, the total number of $a_{i}$ coefficients is 8 and the total number of $b_{i j}$ coefficients is 24 . In the RBOSR algorithm, the regularizing bound $u$ is chosen to be 10. The optimal coefficients $a_{i}^{*}$ and $b_{i j}^{*}$ obtained by the OSR, BOSR, RBOSR are listed in Tables 1, 2, and 3, respectively. These coefficients are also graphically represented by Figures 1,2 , and 3 . It can be observed that the first several weighting coefficients for the OSR are relatively small. As the IMF order increases, the $a_{i}$ coefficients also increase to some values close to one. This can be seen as a generalization of the PAR-EMD in which binary selection on the IMFs is replaced by linear weighting of the IMFs. The result is also in agreement with that of the PAR-EMD where it is found that the lower-order IMFs contain more noise components than the higher-order IMFs. Consequently, lower-order IMFs should be assigned small weights in denoising. When comparing the optimal $b_{i j}$ coefficients obtained by the BOSR and RBOSR, we see that the BOSR yields coefficients that differ in magnitude on the order of thousands (see Table 2 and Figure 2), while the optimal coefficients obtained by the RBOSR are closer to each other (see Table 3 and Figure 3). Therefore, the regularization process mitigates the numerical instability of the original BOSR algorithm.

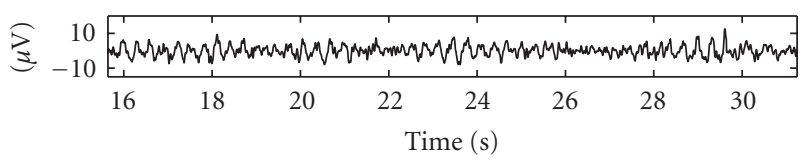

(a)

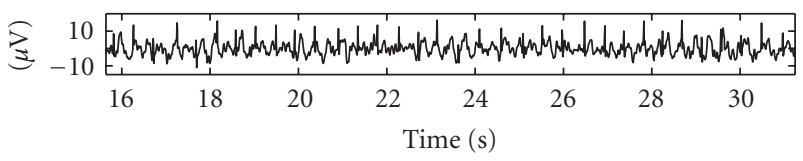

(b)

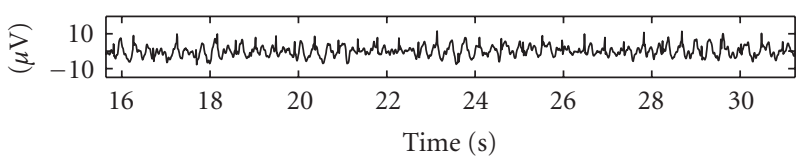

(c)

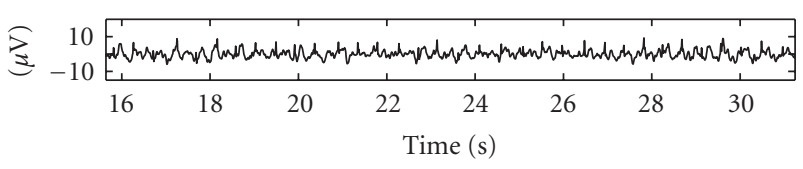

(d)

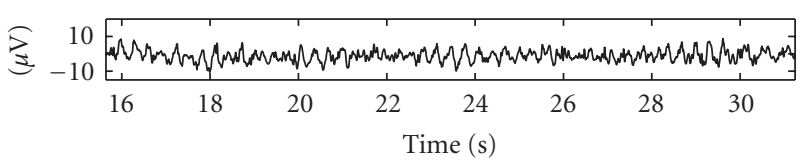

(e)

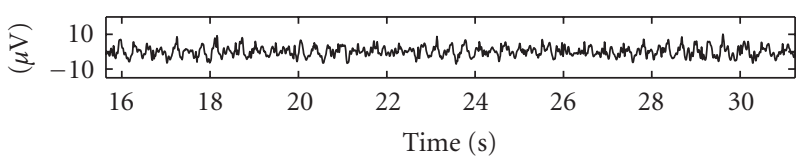

(f)

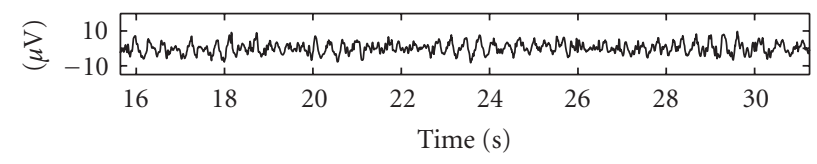

(g)

FIGURE 9: ECG interference removal in EEG. (a) Original EEG, (b) EEG containing ECG interferences, (c) OSR (MSE = 4.1883), (d) adaptive OSR (MSE = 3.3599), (e) BOSR (MSE = 2.7189), (f) adaptive BOSR $(\mathrm{MSE}=2.3354)$, $(\mathrm{g})$ RBOSR $(\mathrm{MSE}=2.0432)$. 
TABLE 3: Optimal coefficients of the regularized BOSR algorithm $(M=1)$.

\begin{tabular}{|c|c|c|c|c|c|c|c|c|}
\hline \multirow{2}{*}{$b_{i j}^{*}$} & \multicolumn{8}{|c|}{ IMF order $i$} \\
\hline & 1 & 2 & 3 & 4 & 5 & 6 & 7 & 8 \\
\hline-1 & 0.2235 & 0.3774 & 0.2196 & 0.3947 & 0.1248 & 0.3133 & -0.8960 & 0.0719 \\
\hline 0 & 0.4651 & 0.3275 & 0.2643 & 0.1128 & 0.5437 & 0.3387 & 0.3509 & 0.3213 \\
\hline 1 & 0.1891 & 0.2102 & 0.4897 & 0.4160 & 0.3245 & 0.3193 & 1.5481 & 0.5592 \\
\hline
\end{tabular}

The denoising results are shown in Figure 4 where we also show the results of the Butterworth lowpass filtering and the PAR-EMD algorithm. The noisy signal is shown in Figure 4(a) in which testing samples from 1000-1200 are shown. Figures 4(b), 4(c), 4(d), 4(e), and 4(f) show the denoised signals reconstructed by the linear filter, PAREMD, OSR, BOSR, and RBOSR, respectively, and compare the resulting signals with the original signal. It can be seen that the OSR, BOSR, and RBOSR produce a signal closer to the original signal than the other two methods. However, the BOSR performs slightly better than the OSR since the residual error is smaller. The reason for the improved performance is that the BOSR takes the signal correlation into account. Furthermore, the performances of the BOSR and RBOSR are very close. This shows that even though the coefficients of the BOSR are much more dispersed than those of RBOSR, the BOSR performance does not suffer from this. Measured quantitatively by the MSE from (35), these algorithms yield MSE of 0.0193 for linear filter, 0.01 for the PAR-EMD, 0.0063 for the OSR, 0.0046 for the BOSR, and 0.0046 for the RBOSR.

We remarked in Section 4 that the bidirectional $b_{i j}$ coefficients act as a FIR filter in the time domain for the $i$ th IMF. Therefore, it is interesting to investigate the behavior of these filters as the order of IMF changes. Starting from the first IMF, we plot the frequency responses of the filters used in the BOSR algorithm in Figure 5. It can be seen that the first filter $B_{1}(\omega)$ applied to IMF 1 exhibits lowpass characteristics. As the IMF order increases, the filters first become bandpass filters and then more highpass-like filters. In the denoising application, the first IMF contains strong noise components. So the filter tries to filter the noise out and leaves only lowpass signal components. For the mid-order IMFs, noise components are mainly located in certain frequency bands, which tunes the filter to be bandpass. For high-order IMFs, the filter gain is high and the DC frequency range is nearly kept unchanged $(0 \mathrm{~dB})$. The BOSR is equivalent to filtering the signal by $N$ different filters in $N$ different IMFs. This will not be possible if we simply use the partial summation of IMFs. The frequency responses of the filters used in the RBOSR are also shown in Figure 6 with a different behavior observed. These filters are either of lowpass or bandpass type and no highpass characteristics are exhibited. Also, the filter gains for RBOSR are generally smaller than those of BOSR, which is a result of coefficient regularization in the optimization process.

A more thorough study using a wide range of different realizations of stochastic signals is carried out by Monte Carlo simulation. Figure 7 shows the MSE versus SNR for the five algorithms: linear filtering, PAR-EMD, OSR, BOSR, and RBOSR. At each SNR, 500 runs are performed to obtain an averaged MSE as shown in the figure. We see that the OSR and BOSR algorithms outperform the linear filtering and PAR-EMD over the entire SNR range. The performances of the BOSR and RBOSR are better than that of the OSR, as expected. The BOSR performs slightly better than the RBOSR even though its coefficients are less regular.

To investigate the effects of the memory length $M$ on the BOSR performance, five different values of $M$ are chosen $(M=1,2,3,4,5)$. Monte Carlo simulation is carried out to compare the performances of the BOSR for different $M s$. From Figure $8(\mathrm{a})$, using larger $M$ does not significantly improve the performance as we see those curves are getting closer to each other as $M$ increases. A zoomed-in view around SNR $=15 \mathrm{~dB}$ in Figure 8(b) more clearly shows that larger $M$ yields lower MSE, though this difference is not easily distinguishable from the larger scale plot. It is therefore advised to choose a small $M$ instead of large $M$ in the BOSR since small $M$ can do as good a job as large $M$ but with less complexity.

Example 2. Electroencephalogram (EEG) is widely used as an important diagnostic tool for neurological disorder. Cardiac pulse interference is one of the sources that affect the EEG recording [18]. The EMD method is especially useful for nonlinear and nonstationary biomedical signals [19-22]. The optimal reconstruction algorithms based on EMD are therefore used to remove the ECG interferences from EEG recording.

Real EEG and ECG recordings are obtained from a 37year-old woman at Alfred I., DuPont Hospital for Children in Wilmington, Delaware. The signals are sampled at $128 \mathrm{~Hz}$. The EEG signal with ECG interferences is obtained by adding attenuated ECG component to EEG, that is, $x(t)=x_{e}(t)+$ $\alpha x_{c}(t)$, where $x_{e}(t)$ is the EEG, $x_{c}(t)$ is the ECG, and $\alpha=0.6$ reflects the attenuation in the pathways. The total duration of recording is about 29 minutes and we select the first 2000 samples (0-15.625 seconds) as the training samples and the next 2000 samples (15.625-31.25 seconds) as the testing samples. The original EEG and the EEG containing ECG interferences are shown in Figures 9(a) and 9(b), respectively. It is clear that the spikes due to the QRS complex of ECG is prominent in EEG. The spectra of ECG and EEG are overlapped because the bandwidth for ECG monitoring is $0.5-50 \mathrm{~Hz}$, while the frequency bands of EEG range from $0.5-$ $13 \mathrm{~Hz}$ and above [23]. Therefore, simple filtering techniques cannot be used to separate EEG from ECG interferences. The three optimal reconstruction methods, OSR, BOSR, and 
RBOSR, together with their adaptive versions, are applied to the ECG contaminated EEG signal. The memory length $M$ is set to 1 for both BOSR and RBOSR and the bound $u$ for RBOSR is chosen to be 10 . The reconstructed samples are shown in Figures 9(c), 9(d), 9(e), 9(f), and 9(g). The resulting signal of the OSR still has some residual spikes. Both BOSR and RBOSR yield signal waveforms that are closer to the original EEG. However, there is a baseline wander in the initial stage of the BOSR result while this baseline wander does not exist in the RBOSR result. Adaptive modes of the OSR and BOSR are used and the results are shown in Figures 9(d) and 9(f), respectively. From these figures, all these optimal reconstruction methods are able to remove the ECG interferences from EEG to some extent. But the BOSR and RBOSR are better than the OSR, which agrees with the first example. In terms of MSE, the OSR has MSE $=4.1883$ while the BOSR and RBOSR achieve MSE of 2.7189 and 2.0432, respectively. The adaptive modes of OSR and BOSR yield MSE of 3.3599 and 2.3354, thus slightly improve the original algorithms.

\section{CONCLUSION}

The empirical mode decomposition is a tool for analyzing nonlinear and nonstationary signals. Conventional EMD, however, does not impose on optimality conditions for reconstruction from IMFs. In this paper, several improved versions of EMD signal reconstruction that are optimal in the minimum mean square error sense are proposed. The first algorithm OSR estimates a given signal by linear weighting of the IMFs. The coefficients are determined by solving a linear set of equations. To consider the temporal structure of a signal, BOSR is then proposed. The weighting of the BOSR is carried out not only in the IMF order direction, but also in the temporal direction. It is able to compensate for the time correlation between adjacent samples. The proposed algorithms are applied to signal denoising problem, where both the OSR and BOSR have better performance than the traditional partial reconstruction EMD. These methods are also applied to real biomedical signals where ECG interferences are removed from EEG recordings. The optimal EMD reconstruction methods proposed in this paper give some new insight to this promising signal analysis tool.

\section{REFERENCES}

[1] N. E. Huang, Z. Shen, S. R. Long, et al., "The empirical mode decomposition and hilbert spectrum for nonlinear and nonstationary time series analysis," Proceedings of the Royal Society A, vol. 454, no. 1971, pp. 903-995, 1998.

[2] P. Flandrin, G. Rilling, and P. Gonçalvés, "Empirical mode decomposition as a filter bank," IEEE Signal Processing Letters, vol. 11, no. 2, part 1, pp. 112-114, 2004.

[3] P. Flandrin and P. Gonçalvés, "Empirical mode decomposition as a data-driven wavelet-like expansions," International Journal of Wavelets, Multiresolution and Information Processing, vol. 2, no. 4, pp. 477-496, 2004.

[4] K. Coughlin and K. K. Tung, "Eleven-year solar cycle signal throughout the lower atmosphere," Journal of Geophysical Research, vol. 109, no. D21, p. D21105, 2004.
[5] J. C. Nunes, S. Guyot, and E. Deléchelle, "Texture analysis based on local analysis of the bidimensional empirical mode decomposition," Machine Vision and Applications, vol. 16, no. 3, pp. 177-188, 2005.

[6] D. Pines and L. Salvino, "Structural health monitoring using empirical mode decomposition and the Hilbert phase," Journal of Sound and Vibration, vol. 294, no. 1-2, pp. 97-124, 2006.

[7] W. Huang, Z. Shen, N. E. Huang, and Y. C. Fung, "Engineering analysis of biological variables: an example of blood pressure over 1 day," Proceedings of the National Academy of Sciences of the United States of America, vol. 95, no. 9, pp. 4816-4821, 1998.

[8] H. Liang, Q.-H. Lin, and J. D. Z. Chen, "Application of the empirical mode decomposition to the analysis of esophageal manometric data in gastroesophageal reflux disease," IEEE Transactions on Biomedical Engineering, vol. 52, no. 10, pp. 1692-1701, 2005.

[9] B. Weng, M. Blanco-Velasco, and K. E. Barner, "ECG denoising based on the empirical mode decomposition," in Proceedings of the 28th Annual International Conference of IEEE Engineering in Medicine and Biology Society (EMBS '06), pp. 14, New York, NY, USA, August-September 2006.

[10] J. C. Nunes, Y. Bouaoune, E. Delechelle, O. Niang, and Ph. Bunel, "Image analysis by bidimensional empirical mode decomposition," Image and Vision Computing, vol. 21, no. 12, pp. 1019-1026, 2003.

[11] R. Balocchi, D. Menicucci, E. Santarcangelo, et al., "Deriving the respiratory sinus arrhythmia from the heartbeat time series using empirical mode decomposition," Chaos, Solitons \& Fractals, vol. 20, no. 1, pp. 171-177, 2004.

[12] N. Huang and N. O. Attoh-Okine, The Hilbert-Huang Transform in Engineering, CRC Press, Boca Raton, Fla, USA, 2005.

[13] B. Weng, M. Blanco-Velasco, and K. E. Barner, "Baseline wander correction in ECG by the empirical mode decomposition," in Proceedings of the 32nd Annual Northeast Bioengineering Conference (NEBC '06), pp. 135-136, Easton, Pa, USA, April 2006.

[14] E. Deléchelle, J. Lemoine, and O. Niang, "Empirical mode decomposition: an analytical approach for sifting process," IEEE Signal Processing Letters, vol. 12, no. 11, pp. 764-767, 2005.

[15] P. Flandrin, P. Gonçalvés, and G. Rilling, "Detrending and denoising with empirical mode decomposition," in Proceedings of the 12th European Signal Processing Conference (EUSIPCO '04), Viena, Austria, September 2004.

[16] H. Akaike, "A new look at the statistical model identification," IEEE Transactions on Automatic Control, vol. 19, no. 6, pp. 716-723, 1974.

[17] D. G. Luenberger, Introduction to Linear and Nonlinear Programming, Addison-Wesley, Reading, Mass, USA, 1973.

[18] J. Sijbersa, J. Van Audekerke, M. Verhoye, A. Van der Linden, and D. Van Dyck, "Reduction of ECG and gradient related artifacts in simultaneously recorded human EEG/MRI data," Magnetic Resonance Imaging, vol. 18, no. 7, pp. 881-886, 2000.

[19] M. Blanco-Velasco, B. Weng, and K. E. Barner, "A new ECG enhancement algorithm for stress ECG tests," in Proceedings of IEEE International Conference on Computer in Cardiology (CIC '06), pp. 917-920, Valencia, Spain, September 2006.

[20] B. Weng, G. Xuan, J. Kolodzey, and K. E. Barner, "Empirical mode decomposition as a tool for DNA sequence analysis from terahertz spectroscopy measurements," in Proceedings of IEEE International Workshop on Genomic Signal Processing and Statistics (GENSIPS '06), pp. 63-64, College Station, Tex, USA, May 2006. 
[21] J. Dauwels, T. M. Rutkowski, F. Vialatte, and A. Cichocki, "On the synchrony of empirical mode decompositions with application to electroencephalography," in Proceedings of IEEE International Conference on Acoustics, Speech and Signal Processing (ICASSP '08), pp. 473-476, Las Vegas, Nev, USA, March 2008.

[22] M. Blanco-Velasco, B. Weng, and K. E. Barner, "ECG signal denoising and baseline wander correction based on the empirical mode decomposition," Computers in Biology and Medicine, vol. 38, no. 1, pp. 1-13, 2008.

[23] R. M. Rangayyan, Biomedical Signal Analysis: A Case-Study Approach, IEEE Press, New York, NY, USA, 2002. 